\title{
The Informational Basis of Social Judgments: Memory for Informative and Uninformative Arguments
}

\author{
YaACOV SCHUL \\ The Hebrew' University of Jerusalem
}

AND

\section{Eugene Burnstein}

The University of Michigan

Received January 12, 1981

\begin{abstract}
This study investigates the encoding and retrieval of arguments in an opinion formation task. It is bascd on a model of opinion formation that partitions the latter process into initial encoding, elaborative encoding, integration, and decision. According to this model elaborative encoding depends on two factors: (i) the informativeness of the arguments and (ii) their thematic relatedness. Since it is reasonably well established that the likelihood of retrieving an argument is an increasing function of the amount of elaboration performed on it, the first hypothesis is straightforward, namely. that the memory for an argument will increase with its informativeness and with its thematic relatedness to other arguments. The second hypothesis assumes that by dint of their closer association with the decision, informative arguments occupy a more central position in the representation of an opinion than uninformative ones. This implies that an informative argument should be accessed and reported prior to an uninformative argument, even when differences in retrievability (i.e., probabilities of recall) are controlled. The findings were consistent with such an analysis.
\end{abstract}

In developing an opinion on an issue a person often must decide among alternative positions, beliefs, solutions, courses of action, etc. An informative argument is one that enables the individual to achieve this end, namely, to make a choice or at least to narrow the range of possibilities, while an uninformative argument is one that does not. This paper examines

The research reported here was supported by a grant from the National Science Foundation (BNS 7915416). Address reprint requests to Eugene Burnstein, Research Center for Group Dynamics, Institute for Social Research, University of Michigan, Ann Arbor, MI 48106. or to Yaacov Schul, Department of Psychology, The Hebrew University of Jerusalem. Jerusalem, Israel. 
the possibility that in opinion formation, arguments are marked in memory according to their informativeness.

Intuitively we know that an informative argument is encoded differently than an uninformative one. For example, suppose one were asked, "Should Mary drive or walk to work?" The argument "She wears glasses to correct near-sightedness" by itself is uninformative inasmuch as it has no direct bearing on the opinion. Hence, we expect that this piece of information will be processed with minimal elaboration, no more than is needed to understand it as a discrete (and tangential) fact. The very same argument, however, becomes highly informative when it appears together with another piece of information, say, "She broke her glasses last night at a party." Now one feels that both propositions ought to be tightly linked and elaborated upon. That is, inferences will be drawn based on prior knowledge about the consequences of driving without glasses (e.g., "Last week Igor, who has the same eye problem, tried to drive without glasses and had a bad accident"), and both propositions and inferences will be represented within a single unified memory structure.

There is, in fact, a decent amount of evidence to support the above point of view: Recent research suggests that arguments that are informative receive more attention (Fiske, 1980), are more accessible (Carlston, 1980; Judd \& Kulik, 1980; Lingle, Geva, Ostrom, Leippe, \& Baumgardner, 1979), and have greater impact on judgments (Burnstein \& Schul, 1982; Fiske, 1980; Tyler, 1980; Vinokur \& Burnstein, 1978) than those that are uninformative-or conversely, when an uninformative argument is encountered during opinion formation, it is comprehended more narrowly, with less elaboration, than an informative one.

Elaboration, of course, does not depend only on the informativeness of an argument. We know that thematically related arguments are automatically associated and elaborated upon independently of their informativeness (e.g., Anderson, 1976; Burnstein \& Schul, 1982; Mandler, 1979; Smith, Adams, \& Schorr, 1978). This means that if arguments are thematically related, they will be linked by a denser network of associations than if they are unrelated. For instance, the statement "Mary wears glasses to correct near-sightedness" will receive greater elaboration when it appears together with the argument "Mary"s father is an optometrist" than when it accompanies the argument "The price of gas is really soaring." In the former case the two arguments are linked to each other via general knowledge about "glasses," "optometrists," "optometrists" daughters," etc. In the latter case, the two propositions are more likely to be represented as discrete pieces of information.

There is, in addition, a less straightforward prediction that can be made about recall in an opinion formation task, namely, that there will be an order effect whereby informative arguments are activated earlier than uninformative ones. To derive this prediction let us describe briefly 
our analysis of the process of opinion formation (Burnstein \& Schul, 1982): We divide opinion formation into several distinct mental operations, namely, initial encoding, elaborative encoding, integration, and decision. During initial encoding the physical representation of the argument undergoes perceptual analysis and segmentation whereby it is transformed into symbolic structures. Elaborative encoding refers to the process by which each argument is linked with general information represented in the form of schemata. As a result, each argument is supplemented by the knowledge contained in its particular encoding schema. The opinion formation task also requires that the person eventually integrate diverse arguments on the topic so that they are represented in a coherent and consistent manner. This means that at some point the separate arguments have to be encoded within one and the same schema. Integration, in turn, prepares the information for the final operation, the decision, whereby the now-integrated arguments are interpreted within an evaluative schema and this evaluation is translated into a response format, such as a 6point scale, a frown, a smile, etc. (See Burnstein \& Schul (1982) for a more detailed description.)

In the present study arguments are processed within a particular context. One important element of this context, the starter question (e.g., "Should Mary drive or walk to work?'), activates a schema for encoding succeeding arguments (e.g., "She broke her glasses last night at a party"). The elaborations made during encoding (e.g., "Last week Igor, who has the same eye problem, tried to drive without his glasses and had a bad accident") provide additional contextual elements. Eventually the arguments and the elaborations made on them are integrated to arrive at a decision (e.g., "She ought to walk!"), which itself then becomes a significant contextual element in the representation (see below). In propositional network models such as Anderson and Bower (1972, 1973) contextual elements in general are termed list markers and refer to information activated during encoding that designate items as belonging to a specific set. There is an important class of markers that represent the gist of the items within a set. These are called headers or prototype markers. They identify the set as a whole and differentiate it from other sets.

We will assume that the representation of the decision, that is, the opinion or judgment, serves as a prototype marker for the set of arguments underlying it. Burnstein and Schul (1982) provided some evidence to show that the decision is a functionally distinct operation in opinion formation. Evidence that the decision has a distinct representation in memory (vis à vis trait information) was obtained by N. Anderson and associates using the methods of "cognitive algebra" (Anderson \& Hubert, 1963; Anderson \& Farkas, 1973; Riskey, 1979). Additional support for the decision being stored separately was presented by Posner and Snyder 
(1975). They concluded that impression formation produces "two memory structures, one consisting of a list of item names [the traits], and the other consisting of a general emotional response to the items" (pp. 7980). Moreover, studies using semantic material other than trait descriptions generally indicate that when information is integrated its gist is abstracted and stored separately as a header (or tag-node) of the larger structure (Hayes-Roth, 1977; Hebb, 1949; Reder \& Anderson, 1980). Finally, there are recent findings (e.g., Lingle \& Ostrom, 1979; Ebbesen, 1980; Schul, 1981) suggesting that in an impression formation task the decision may be retrieved independent of the trait information.

During recall, according to Anderson and Bower, reinstatement of context (i.e., presentation of the starter question) activates the prototype marker (i.e., the decision). A search is then carried out from this node extending along the marked paths. These are associations labeling arguments as belonging to the same opinion domain. When a node is reached with no outgoing paths that are marked (i.e., the arguments accessible from this node are not recognized as falling within the opinion domain), another list marker is activated and the search resumes.

As a last step in our analysis of recall order and informativeness, consider that two items will be associated in memory to the extent that there is an inferential (or semantically "causal") relationship between them (Dooling \& Christiaansen, 1977; Reder, 1979; Spiro, 1977; Tversky \& Kahneman, 1978). Thus we assume a marked path between a decision and the arguments that "caused" it: When the starter question ("Should Mary drive or walk to work?") initiates recall, individuals will tend first to think of the opinion header, that is, their decision ("She ought to walk!"), and then to think of informative arguments, that is, the reasons for the decision ("She broke her glasses"). Uninformative arguments will be recalled only later when there is a marked path between their node and that of an informative argument (e.g., a common theme) or when they are associated with a (nonprototype) opinion marker that is not activated until the marked paths radiating from the prototype marker have been exhausted. Thus, the Anderson-Bower model suggests that informative arguments occupy a central position, and uninformative arguments, a peripheral position in the representation of an opinion. ${ }^{1}$

To summarize, elaborative encoding of arguments is assumed to depend on two factors: (i) the informativeness of the arguments, and (ii) their thematic relatedness. It is reasonably well established that the likelihood of retrieving an argument is an increasing function of the amount of elaboration performed on it. Therefore, our first hypothesis implies that

Comparable analyses demonstrating the importance of the central-peripheral distinction for chacterizing informative and uninformative arguments in the area of attitude change and persuasion can be found in Fishbein and Ajzen (1975), Peak, (1958), Rosenberg and Abelson. (1960), Wishner, (1960), and Zajonc. (1960). 
the memory for an argument will increase with its informativeness and with its thematic relatedness to other arguments. Our second hypothesis assumes that by dint of their close association with header or prototype information, in particular the decision, informative arguments occupy a more central position than uninformative arguments. Hence, informative arguments should be accessed and reported prior to uninformative arguments, even when differences in retrievability (i.e., probabilities of recall) are controlled.

\section{METHOD}

\section{Overview}

The study consisted of two parts. In the first part subjects were given a series of hypothetical "situations." These were presented in the form of a starter question followed by five arguments that varied in their informativeness and in their thematic relatedness. Subjects expressed an opinion about each situation on the basis of these arguments. During the second part, subjects were given a surprise cued recall test where they were presented with the starter questions and were asked to recall the arguments associated with them.

\section{Subjects}

Thirty-six undergraduates at the University of Michigan participated in the experiment as a partial fulfillment of their introductory psychology course requirement.

\section{Apparatus}

Subjects were seated in individual booths, equipped with a 12 -in. monitor and a 6 -button response box (buttons labeled from " 1 " to " 6 "). Presentation of the stimuli was controlled by a PDP-11 computer system with responses and reaction times recorded under a program's control.

\section{Stimuli}

The first part consisted of 18 segments. In each of them subjects were presented with a dilemma-like situation consisting of a starter question and five arguments. The arguments varied in their informativeness and/or their relatedness to another argument. Specifically, there were four categories of arguments:

(a) arguments that were informative in regard to the decision and were thematically related to another argument (I-R type arguments);

(b) arguments that were informative but not related to another argument (I-NR type arguments);

(c) arguments that were related to another argument but not informative in regard to the decision (NI-R type arguments);

(d) arguments that were neither informative nor related to another argument (NI-NR type arguments).

One of the starter questions and its attendant arguments is shown in Table 1. The other seven experimental segments (see design section) were cued by the following:

(1) "Which graduate school should Mike go to, A or B?"

(2) "Tim is a wealthy investor who must decide whether to finance project G or H."

(3) "Should Bob live in apartment housc Q or apartment house P?"

(4) "Should Jane go to a movie or stay home and study?"

(5) "Should Ron shop at Stop-N-Shop or Fastway?"

(6) "Ray must decide between two cars, either a Champion or a Roadster."

(7) "Which job should Kathy chosc, X or Y?" 


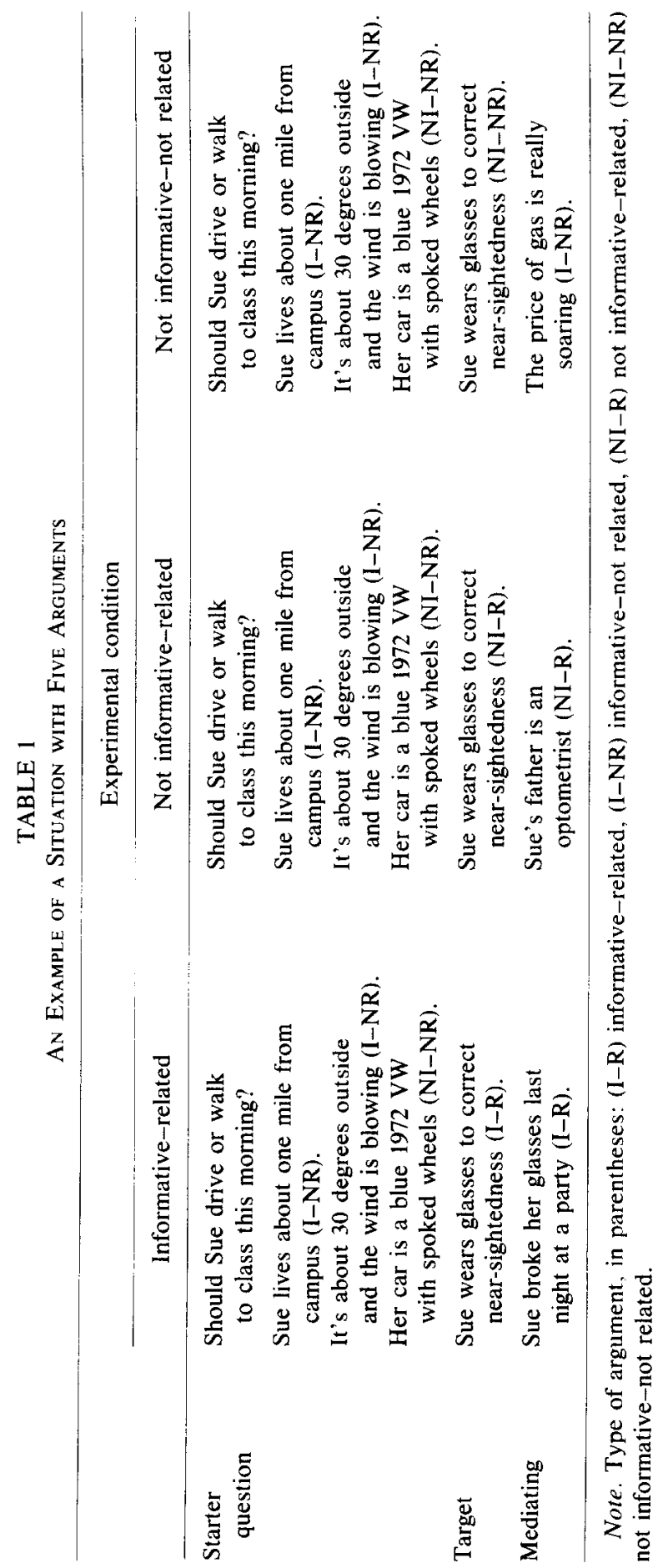




\section{Design}

Out of the 18 segments, 2 were used for practice and 8 were used as fillers. They contained arguments from the above four categories and were the same for all subjects. The remaining eight segments will be termed the experimental segments. In each of the experimental segments, the situation and four of the arguments associated with it were identical for all subjects. Two of these arguments were I-NR and one was NI-NR (see Table 1). The relatedness of the fourth argument (the target argument, hereafter) to the fifth argument varied between conditions as a function of the latter (the mediating argument. hereafter). The three experimental conditions (a between subject factor) differ in respect to the mediating argument, and thereby in respect to the status of the target argument. Note that the content of the target argument remained the same regardless of its status. The three conditions are labeled according to the status of the target argument.

In the informative-related condition, the mediating argument was not only thematically linked to the target argument but it also made the latter informative in respect to the decision; in the uninformative-related condition, the mediating argument was related to the target argument, but the two were not relevant to the opinion task-they were uninformative: finally, in the uninformative-unrelated condition the mediating argument was not related to the target argument, nor was the latter informative.

\section{Procedure}

Subjects participated in groups ranging in size from one to six. Upon arriving at the laboratory, they were randomly assigned to an experimental condition (with the constraint that there be an equal number per condition) and seated in an individual booth. After reading the instructions, they responded to two practice segments that acquainted them with the monitor, the response box, and the experimental procedure. After the two practice segments were completed, the experimenter answered questions and the subjects were presented with the remaining 16 segments.

The presentation structure of each segment was as follows:

(a) The sentence describing the situation, that is, the starter question, appeared on the monitor for a fixed time (.6 sec per word) and was erased.

(b) The five arguments were then presented one at a time (.4 sec per word), with a 2sec interval between arguments.

(c) Next. the starter question was again displayed on the screen along with a 6-point scale. The end points of the scale were marked with the "situation" alternatives (e.g. "Walk to school," "Drive to school"). The subjects then responded by pressing one of the buttons on the response box. These responses reflected the subject's opinion on which alternative to choose. The presentation order of the segments as well as the presentation order of arguments within a segment were randomized differently for each subject.

Once this part was completed, subjects were given a surprise recall test. This was done in a booklet that presented the eight experimental plus four filler "situations" in a fixed order. Every page of this booklet contained two starter questions, each of which was followed by five blank lines. Subjects were asked to list as many arguments for each situation as they could recall.

\section{RESULTS AND DISCUSSION}

Recall data were coded by three judges. An item was said to be correctly recalled if it rephrased the original (i.e., contained its gist). It was coded as incorrect otherwise (i.e., either it was not recalled at all, it was recalled inaccurately, or it was recalled in the wrong "situation"'). The three judges agreed on $90 \%$ of the cases. In $99 \%$ of the cases there was a majority (i.e., two judges agreed). The remaining cases consisted 
of items in which the judges could not classify according to the original argument, and therefore, they were discarded. Analyses of cases in which a majority of the judges agreed were performed separately from those in which all three judges agreed. There were no appreciable differences between these two sets of analyses, and only the former will be reported.

\section{Accuracy of Recall}

First, we examined the accuracy with which the different types of arguments were recalled. For each subject we computed the accuracy (i.e., percent correct) with which he or she recalled each type of argument across the eight experimental segments. It is important to keep in mind that regardless of the experimental condition, every subject received three identical arguments in each segment (see Table 1), two of which were informative but not related (I-NR) and one that was neither informative nor related (NI-NR). A two-way ANOVA (experimental condition $\times$ argument type) was performed on the accuracy rates with which these arguments were recalled. There was no reliable difference between the three experimental conditions $(F(2,33)<1)$, nor was there a reliable interaction between argument type and experimental condition $(F(2,33)$ $=2.64, p>.08$ ). As hypothesized, however, informative arguments were recalled better than uninformative ones (.65 and .48 , respectively), $F(1,33)=29.42, p<.01$.

In order to determine whether the relatedness of an argument facilitates its retrieval, we examined the accuracy with which the mediating arguments were recalled. A one-way ANOVA revealed that there were reliable differences between the three experimental conditions $(F(2,33)=6.97$, $p<.01)$. Informative and related (I-R) arguments were recalled only marginally better than informative and unrelated (1-NR) arguments $(.80$ vs .65 , respectively), $t(33)=1.67, p<.1$. At the same time, the I-NR arguments were recalled significantly better than NI-R arguments (.45), that is, those that were related but not informative, $t(33)=2.12, p<$ .05 .

The above analysis confounds the type of argument with its semantic content, although there is no reason to suspect that this produced any systematic bias. In any case, an analysis of the target argument enables us to eliminate this confound. Remember that the informativeness and/or relatedness of a target argument were manipulated via its mediating argument. Therefore, this analysis involves comparisons among identical target arguments with identical retrieval cues. A one-way ANOVA was performed on the accuracy with which subjects recalled the target arguments. It revealed a reliable difference between the three experimental conditions $(F(2,33)=9.90, p<.01)$. Subjects in the informative-related condition recalled the target arguments better than those in the uninformative-related condition (.88 vs .64 , respectively), $t(33)=2.74, p<$ 
.05. Subjects in the latter condition, in turn, recalled the target argument better than those in the uninformative-unrelated condition (.50), $t(33)$ $=1.79, p<.01$. To summarize, (i) informative arguments are more likely to be retrieved than uninformative arguments; (ii) related arguments are more likely to be retrieved than unrelated arguments; and finally, (iii) it appears that informativeness may have a stronger effect than relatedness on the retrievability of arguments. However, the question of how informativeness and relatedness interact to determine accessibility cannot be answered on the basis of the present design.

\section{Clustering in Recall}

In order to obtain further support for the hypothesis that informativeness and relatedness affect the elaboration a proposition receives, we compared the degree of association between the target and the mediating arguments under our three experimental conditions. Elaborative encoding in the present context involves the creation of associative links among the propositions in the communication as well as links between the latter and schematic knowledge. The extent to which this operation is performed should be reflected in the strength of association between the arguments. If the two arguments are highly associated, that is, clustered in memory, then the recall of one should be accompanied by the recall of the other. In other words, either both arguments are accessed, or neither is accessed. If, however, they are not associated, the recall of one argument should be independent of the recall of the other.

For each subject the rho correlation coefficient (Bishop, Fienberg, \& Holland, 1975) was computed to estimate the association in the recall of the target and the mediating arguments over the eight "situations." A one-way ANOVA was performed on these coefficients to determine whether they varied as a function of the experimental condition. ${ }^{2}$ The analysis revealed that the three conditions reliably differed from each other $(F(2,27)=7.04, p<.01)$. Specifically, when the arguments were both informative and related they were more highly associated than when they were related but not informative (rhos .57 and .23 , respectively, $t(27)=1.98, p<.06)$. Moreover, arguments were least associated when they were neither informative nor related (rho $=-.06$ ). 'This suggests that the effects of informativeness and thematic relatedness are combined to produce a more accessible representation than either separately. Thus, the hypothesis that the claboration an argument receives increases with its informativeness and with its thematic relatedness to other arguments in the communication is reasonably well supported.

2 Four subjects in the IR condition and one in each of the other two conditions had to be dropped from the analysis because they recalled all the target argument perfectly (and hence the rho could not have been computed). 


\section{Priority in Recall}

According to our analysis, not only should informative arguments be recalled better than uninformative ones, but they should also be accessed prior to them. Typically, there is a substantial positive correlation between the probability that an argument will be recalled and its serial position in recall. Our hypothesis, however, is that informative arguments will have priority of entry even when the probability of recall is controlled. In contrast, the relatedness of the arguments should not affect its priority of entry once recall probability is controlled. Ideally, these hypotheses should have been tested simultaneously in a two-way ANOVA with informativeness and relatedness as the independent variables. Since the design of the present experiment does not permit such an analysis, each of these hypotheses had to be examined separately (but see below).

For each subject we computed a set of difference scores, each corresponding to a difference between the proportion of cases an informative argument was reported prior to an uninformative one, and the proportion of cases the uninformative argument was reported prior to the informative one. This was done only in cases when both informative and uninformative arguments were recalled (so that the probability of recall is controlled). These differences were averaged per subject across all informative/uninformative pairs to yield a priority index $(\mathrm{Pr})$. Note that this index of priority is computed across the two levels of relatedness. ${ }^{3}$ If informative arguments are not accessed prior to uninformative ones, $\mathrm{Pr}$ should not be different from zero. In fact, mean $\operatorname{Pr}$ was $.13(t(35)=$ $2.02, p=.05$ ), indicating that informative arguments tend to be accessed prior to uninformative arguments, even when the likelihood of recall is controlled.

In order to examine the effects of relatedness on the priority of entry, we repeated the above analysis, that is, we computed a second priority index, but this time on the basis of relatedness rather than informativeness. If related arguments have priority in recall over unrelated ones, the results of this analysis should have been similar to those in the previous analysis. In fact, relatedness did not have a statistically significant effect $(\operatorname{Pr}=.04, t(35)=.8)$.

Finally, even though it was not possible to perform a complete two way ANOVA (relatedness $x$ informativeness), we compared the effects of arguments types within each experimental condition (cf. Table 1) on the priority of informative arguments in recall. Due to the small number of observations in each analysis, none of the differences reached acceptable levels of significance. Nonetheless, they were all in the predicted direction.

${ }^{3}$ In fact, this analysis may be biased against our hypotheses. Note that there are more I-NR arguments than NI-NR arguments. This means that the likelihood of an argument being difficult to access because it is unrelated is greater for informative than for uninformative arguments. Since accessibility and recall order are correlated, it follows that even when both are recalled, uninformative arguments should be reported prior to informative arguments. 


\section{CONCLUSIONS}

An opinion formation task demands that individuals elaborate upon a list of arguments, integrate the resultant knowledge into a unitary representation, and come to a decision. The decision functions as a header or prototype marker that tags the arguments for pertinence, namely, their usefulness in arriving at an opinion. We have called such arguments informative. Our analysis suggested that informative arguments lend themselves to elaborative encoding to a larger extent than uninformative arguments. Hence, informative arguments will be accessed more readily than uninformative arguments. In addition, informative arguments, unlike uninformative ones, have a semantically "causal" or inferential relationship to the decision and thereby should be closely associated with the decision in memory. If the decision is in fact a prototype marker, it will be activated as soon as the person searches the particular opinion domain for arguments. As a result, informative arguments should be accessed prior to uninformative arguments. Our findings support such an analysis. First, memory for informative arguments was superior to that for uninformative arguments. Second, when the probability of recall was controlled, informative arguments were retrieved prior to uninformative ones.

\section{REFERENCES}

Anderson, J. R. FRAN: A simulation model of free recall. In G. H. Bower (Ed.), The psychology of learning and motivation (Vol. 5). New York: Academic Press, 1972.

Anderson, J. R. Language, memory, \& thought. Hillsdale, N. J.: Erlbaum, 1976.

Anderson, J. R., \& Bower, G. H. Human associative memory. Washington. D. C.: Winston \& Sons, 1973.

Anderson, J. R., \& Bower, G. H. Recognition and retrieval processes in free recall. Psychological Review, 1972, 79, 97-123.

Anderson, N. H., \& Farkas, A. J. New light on order effects in attitude change. Journal of Personality \& Social Psychology, 1973, 28, 88-93.

Anderson, N. H., \& Hubert, S. Effect of concomitant verbal recall on order effects in personality impression formation. Journal of Verbal Learning and Verbal Behavior. 1963, 2, 379-391.

Bishop, Y. M., Fienberg, S. E., \& Holland, P. W. Discrete multivariate analysis. Cambridge, Mass.: MIT Press, 1975.

Burnstein. E., \& Schul, Y. The informational basis of social judgments: The operations in forming an impression of another person. Journal of Experimental Social Psychology, 1982, 18, 217-234.

Carlston, D. E. The recall and use of traits and events in social inference processes. Journal of Experimental Social Psychology, 1980, 16, 303-328.

Craik, F. I. M., \& Tulving, E. Depth of processing and the retention of words in episodic memory. Journal of Experimental Psychology, 1975, 104, 268-294.

Dooling, D. J., \& Christiaansen, R. E. Episodic and semantic aspects of memory for prose. Journal of Experimental Psychology: Human Learning and Memory, 1977, 3, 428436.

Ebbesen. E. B. Cognitive processes in understanding ongoing behavior. In R. Hastie et al. (Eds.), Person memory: The cognitive basis of social perception. Hillsdale, N. J.: Erlbaum, 1980. 
Fishbein, M., \& Ajzen, I. Belief, attitude, intention, and behavior: An introduction to theory and research. Reading, Mass.: Addison-Wesley, 1975.

Fiske, S. T. Attention and weight in person perception: The impact of negative and extreme behavior. Journal of Personality and Social Psychology, 1980, 38, 889-906.

Hayes-Roth, B. Evolution of cognitive structures and processes. Psychological Revien, $1977,84,260-278$.

Hebb, D. O. The organization of behavior. New York: Wiley, 1949.

Jacoby, L. L., \& Craik, F. I. M. Effects of elaboration of processing at encoding and retrieval: Trace distinctiveness and recovery of initial context. In L. S. Cermak \& F. I. M. Craik (Eds.), Levels of processing in human memory. Hillsdale, N. J.: Erlbaum, 1979.

Judd, C. M., \& Kulik, J. Schematic effects of social attitudes on information processing and recall. Journal of Personality and Social Psychology, 1980, 38, 889-906.

Lingle, J. H., Geva. N., Ostrom, T. M., Leippe, M., \& Baumgardner, M. H. Thematic effects of person judgement on impression organization. Journal of Personality and Social Psychology, 1979, 37, 674-687.

Lingle, J. H. . \& Ostrom. T. M. Retrieval selectivity in memory based impression judgments. Journal of Personality and Social Psychology, 1979, 37, 180-194.

Mandler, G. Organization and repetition: Organizational principles with special reference to rote learning. In L. G. Nilsson (Ed.), Perspectives on Memory Research, Hillsdale, N. J.: Erlbaum, 1979.

Peak, H. Psychological structure and psychological activity. Psychological Review, 1958, 65, 325-347.

Posner, M. I., \& Snyder, C. R. Attention and cognitive control. In R. L. Solo (Ed.), Information processing and cognition: The Loyola symposium. Hillsdale, N. J.: Erlbaum. 1979.

Reder, L. M. The role of elaboration in memory for prose. Cognitive Psychology, 1979, 11, 221-234.

Reder, L. M., \& Anderson, J. R. A partial resolution of the paradox of interference: The role of integrating knowledge. Cognitive Psychology, 1980, 12, 447-472.

Riskey, D. R. Verbal memory processes in impression formation. Journal of Experimental Psychology: Human Learning and Memory, 1979, 5, 271-281.

Rosenberg, M., \& Abelson, R. An analysis of cognitive balancing. In M. Rosenberg et al. (Eds.), Attitude organization and change. New Haven, Conn.: Yale Univ. Press, 1960.

Schul, Y. Integration and abstraction in impression formation. Unpublished doctoral dissertation, University of Michigan, 1981.

Smith, E. E., Adams, N., \& Schorr, D. Fact retrieval and the paradox of interference. Cognitive Psychology, 1978, 10, 438-464.

Spiro. R. J. Constructing a theory of reconstructive memory: The state of the schema approach. In R. C. Anderson, R. J. Spiro. \& W. E. Montague (Eds.), Schooling and the acquisition of knowledge. Hillsdale. N. J.: Erlbaum, 1977.

Tversky, A., \& Kahneman, D. Causal schemata in judgments under uncertainty. In M. Fishbein (Eds.), Progress in social psychology. Hillsdale, N. J.: Erlbaum, 1978.

Tyler, T. R. Impact of directly and indirectly experienced events: The origin of crime related judgments and behaviors. Journal of Personality and Social Psychology, 1980. 39, 13-28.

Vinokur, A.. \& Burnstein, E. Novel argumentation and attitude change: The case of polarization following group discussion. European Journal of Social Psychology, 1978. 8, 335-348.

Wishner, J. Reanalysis of "impression of personality." Psychological Review, 1960, 67, 96-112.

Zajonc, R. B. The process of cognitive tuning in communication. Journal of Abnormal and Social Psychology, 1960, 61, 159-168. 